\title{
Business intelligence as a decision support system tool
}

\author{
O. Kultygin ${ }^{1 *}$, I. Lokhtina ${ }^{1}$ \\ 1 Synergy University, Moscow, Russia \\ *OKultygin@synergy.ru
}

\begin{abstract}
The relevance of the topic considered in the article is to solve the problems of designing management decision support systems for enterprises based on business analytics technology. The research purpose is to analyze the applied methodologies during the design stage of the enterprise information system, to develop principles for using management decision support systems based on business intelligence. The problem statement is to analyze the technologies available on the market, which deal with business analyst systems, their potential use for decision support systems, and to identify the main stages of business analyst for enterprises. Business intelligence (BI) is information that can be obtained from data contained in the operational systems of a firm, enterprise, corporation, or from external sources. The BI can help the management of a company make the best decision in the chosen sphere of human activity faster, and, consequently, win the competition in the market for goods and services. A decision support system (DSS) which uses business intelligence, is an automated structure designed to assist professionals in making decisions in a complex environment and to objectively analyze a subject area. The decision support system is the result of the integration of management information systems and database management systems (DBMS). The internal development of $\mathrm{BI}$ is more cost-effective. The methods used are Structured Analysis and Design Technique and Object-oriented methods. The results of the research: the analysis of the possibilities was conducted and recommendations relating to the use of $\mathrm{BI}$ within DSS were given. Competition between BI software in business analysts reduces the cost of products created making them accessible to end-users - producers, traders and corporations.
\end{abstract}

Keywords: data analysis, external data sources, data transformation, data marts, multidimensional OLAP, MS Analysis Services, decision support system, DBMS

For citation: Kultygin 0., Lokhtina I. Business intelligence as a decision support system tool. Prikladnaya informatika=Journal of Applied Informatics, 2021, vol.16, no.1, pp.52-58. DOI: 10.37791/2687-0649-2021-16$1-52-58$ 


\title{
Бизнес-аналитика как инструмент системы поддержки принятия решений
}

\author{
О. П. Култыгин ${ }^{1 *}$, И. В. Лохтина ${ }^{1}$ \\ 1 Университет «Синергия», Москва, Россия \\ *OKultygin@synergy.ru
}

\begin{abstract}
Аннотация. Актуальность рассматриваемой в статье темы состоит в решении проблем проектирования систем поддержки принятия управленческих решений для предприятий на основе технологии бизнес-аналитики. Цель исследования - проанализировать применяемые методологии на стадии проектирования информационной системы предприятия, разработать принципы использования систем поддержки принятия управленческих решений на основе бизнес-аналитики. Краткая постановка задачи состоит в анализе имеющихся на рынке технологий работы с системами бизнесаналитики и возможности их использования для систем поддержки принятия решений, в выявлении основных стадий работы с бизнес-аналитикой для предприятий. Бизнесаналитика (business intelligence, BI) - это информация, которая может быть получена из данных, содержащихся в оперативных системах фирмы, предприятия, корпорации или во внешних для нее источниках. Бизнес-аналитика может помочь руководству фирмы быстрее принять лучшее решение в выбранной сфере человеческой деятельности, а значит - победить в конкурентной борьбе на рынке товаров и услуг. Система поддержки принятия управленческих решений (СППР) (decision support system, DSS), использующая бизнес-аналитику, - это автоматизированный комплекс, предназначенный для помощи специалисту, принимающему решение в сложных условиях, для объективного анализа предметной области. Система поддержки принятия решений явилась результатом интеграции управленческих информационных систем и систем управления базами данных (СУБД). Разработка системы бизнес-аналитики (ВІ) собственными силами является экономически более эффективной. Используемые методы - методы структурнофункционального анализа и проектирования, методы объектно-ориентированного проектирования. Полученные результаты: проведен анализ возможностей и даны рекомендации использования бизнес-аналитики для создания системы поддержки принятия решений предприятия. Конкуренция производителей программного обеспечения в сфере бизнес-аналитики удешевляет создаваемые продукты, делая их доступными для конечных пользователей - производственных и торговых предприятий и корпораций.
\end{abstract}

Ключевые слова: анализ данных, внешние источники данных, преобразование данных, витрины данных, многомерные OLAP, MS Analysis Services, система поддержки принятия решений, СУБД

Для цитирования: Култыгин О. П., Лохтина И. В. Business intelligence as a decision support system tool // Прикладная информатика. 2021. Т. 16. № 1. С. 52-58. DOI: 10.37791/2687-0649-2021-16-1-52-58

\section{Introduction}

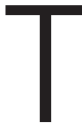
he management decision support systems for enterprises, firms, and corporations are created on the basis of Business Analyst Complexes and relational database management systems (DBMS).
These systems are the core of the integrated information systems of large enterprises or organizations. The proper design of data warehouses is extremely important, which provides convenience for the work of executives: executives, financial analysts, accountants. 